\title{
Clear Cell Carcinoma of the Cervix With OHVIRA Syndrome: A Rare Case Report
}

\author{
Yasuhito Tanase $^{\mathrm{a}, \mathrm{d}}$, Hiroshi Yoshida ${ }^{\mathrm{b}}$, Tomoaki Naka ${ }^{\mathrm{b}}$, Shoichi Kitamura ${ }^{\mathrm{a}}$, Takashi Natsume ${ }^{\mathrm{a}}$, \\ Mayumi Kobayashi Kato a, Masaya Uno a, Mitsuya Ishikawa a , Ahmed Ali Mahmoud Alic, \\ Tomoyasu Kato
}

\begin{abstract}
Obstructed hemivagina and ipsilateral renal agenesis (OHVIRA) syndrome is a rare Mullerian duct anomaly characterized by an obstructed hemivagina, ipsilateral renal agenesis, and uterine didelphys. There are only a few published case reports of OHVIRA syndrome, and cases of OHVIRA syndrome associated with cancer have rarely been reported. In fact, there is only one published report of a case with clear cell carcinoma (CCC) of the cervix. Here, we report a case of CCC of the cervix with OHVIRA syndrome that underwent abdominal radical hysterectomy; we also provide a short literature review of this topic. A 52-year-old woman presented with abnormal vaginal bleeding for 1 month 2 years after menopause. A pelvic examination and preoperative imaging showed uterine didelphys, an obstructed hemivagina with a mass measuring approximately $2 \mathrm{~cm}$ located in her left cervix, and an absence of her left kidney. A colposcopy biopsy reported CCC of the cervix. Clinical staging classified her with stage IB1 disease. Abdominal radical hysterectomy was performed. Her left ectopic ureter led to the left cervix and opened in the endometrium, resulting in a so-called ectopic ureter. Macroscopic examination of the excised specimens showed two cervixes, two corpora of the uterus, and a tumor measuring $1.0 \times 2.0 \mathrm{~cm}$ on the left cervix. In addition to typical OHVIRA symptoms including uterine didelphys, obstructed hemivagina, and renal agenesis, several anatomical variants were present. The current case included those variants as well as an atrophic kidney with an ectopic ureter to the obstructed hemivagina. Based on the results of our case, clinicians should be aware of the risks of cancer and anatomical variants associated with OHVIRA syndrome.
\end{abstract}

Manuscript submitted February 4, 2021, accepted February 19, 2021

Published online February 24, 2021

aDepartment of Gynecology, National Cancer Central Hospital, 5-1-1, Tsukiji, Chuo-ku, Tokyo 104-0045, Japan

bDepartment of Diagnostic Pathology, National Cancer Central Hospital, 5-11, Tsukiji, Chuo-ku, Tokyo 104-0045, Japan

'Department of Obstetrics and Gynaecology, Faculty of Medicine, Assiut University, 71515 Assiut, Egypt

${ }^{\mathrm{d} C o r r e s p o n d i n g ~ A u t h o r: ~ Y a s u h i t o ~ T a n a s e, ~ D e p a r t m e n t ~ o f ~ G y n e c o l o g y, ~ N a t i o n-~}$ al Cancer Central Hospital, 5-1-1, Tsukiji, Chuo-ku, Tokyo 104-0045, Japan. Email: yatanase@ncc.go.jp

doi: https://doi.org/10.14740/wjon1362
Keywords: OHVIRA syndrome; Mullerian duct anomaly; Clear cell carcinoma of the cervix

\section{Introduction}

Obstructive hemivagina and ipsilateral renal agenesis $(\mathrm{OH}-$ VIRA) syndrome, also known as Herlyn-Werner-Wunderlich syndrome, is a rare complex urogenital malformation of the Mullerian ducts. Purslow first reported a case of obstructed hemivagina associated with ipsilateral renal anomalies and uterine anomalies in 1922 [1]. Herlyn and Werner reported a case of the simultaneous occurrence of an open Gardner duct cyst, a homolateral aplasia of the kidney, and a double uterus in 1971 [2], and Wunderlich reported a case of genital malformation with aplasia of the kidney in 1976 [3]. In 2007, Smith et al proposed the concept of OHVIRA to provide a nomenclature for describing this syndrome [4]. This syndrome is characterized by uterus didelphys, which is a rare congenital complex urogenital malformation. The pathogenesis of this syndrome is associated with anomalies in the development of Mullerian and Wolffian ducts. Abnormal development of the Wolffian ducts leads to unilateral renal agenesis and an imperforated hemivagina, and fusion failure of the Mullerian ducts leads to uterine didelphys and bicollis $[5,6]$. Although the pathogenesis of these case reports is considered the same, some anatomical variants associated with OHVIRA syndrome have been reported $[7,8]$.

Given that there are only a few published case reports, the incidence of OHVIRA syndrome remains unknown. Moreover, a case of OHVIRA syndrome associated with cancer has rarely been reported, and only one previous case with clear cell carcinoma (CCC) of the cervix has been reported [9]. Here, we report a case of CCC of the cervix with OHVIRA syndrome that underwent abdominal radical hysterectomy; we also provide a short literature review.

\section{Case Report}

A 52-year-old nulligravida woman presented with 1 month of intermittent vaginal spotting 2 years after menopause. She 


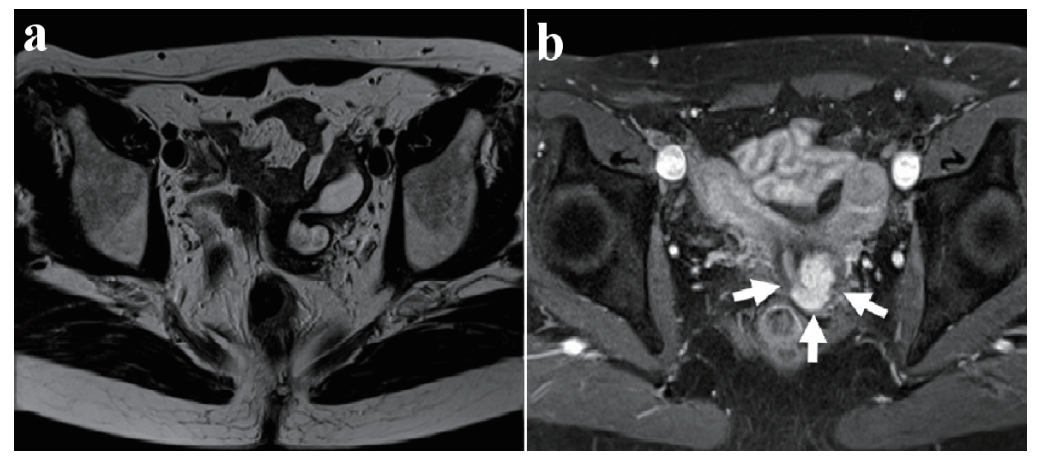

Figure 1. Axial view of pelvic magnetic resonance imaging (MRI). T2-weighted MR image shows the left obstructed hemivagina (a). T2-weighted postcontrast MR image shows uterine didelphys and a tumor measuring $1.0 \times 2.0 \mathrm{~cm}$ on the left side of the uterine cervix (white arrows) (b).

had no history of smoking or medical diseases. Her family history included an uncle who died of gastric cancer. The patient had no history of in-utero diethylstilbestrol (DES) exposure. A previous cervical smear was unremarkable 11 months ago. Speculum examination revealed a single cervix and a $2-\mathrm{cm}$ mass on the cervix, which showed macrocarcinoma of the cervix. No parametrial or vaginal involvement was detected. The biopsy specimen from the tumor on the uterine cervix revealed CCC. The tumor was comprised of atypical cells displaying enlarged nuclei with prominent nucleoli and clear-to-eosinophilic cytoplasm; the cells also displayed papillary structures with a hobnail-like appearance. Immunohistochemical staining showed that the tumor cells were positive for HNF-1 $\beta$ and napsin A. Laboratory data revealed that the patient's squamous cell carcinoma antigens, carbohydrate antigen (CA) 125 levels, and CA19-9 levels were negative. Magnetic resonance imaging (MRI) of her pelvis showed uterine didelphys and a mass measuring $1.5 \times 2.0 \mathrm{~cm}$ located in the left endocervix that had not invaded the parametria, adjacent organs, or regional lymph nodes. Furthermore, a left-sided obstructed hemivagina was observed (Fig. 1). Although an enhanced computed tomography (CT) scan of the chest and abdomen did not reveal evidence of lymph node metastasis or distant metastasis, left renal agenesis was observed (Fig. 2). Based on the findings of

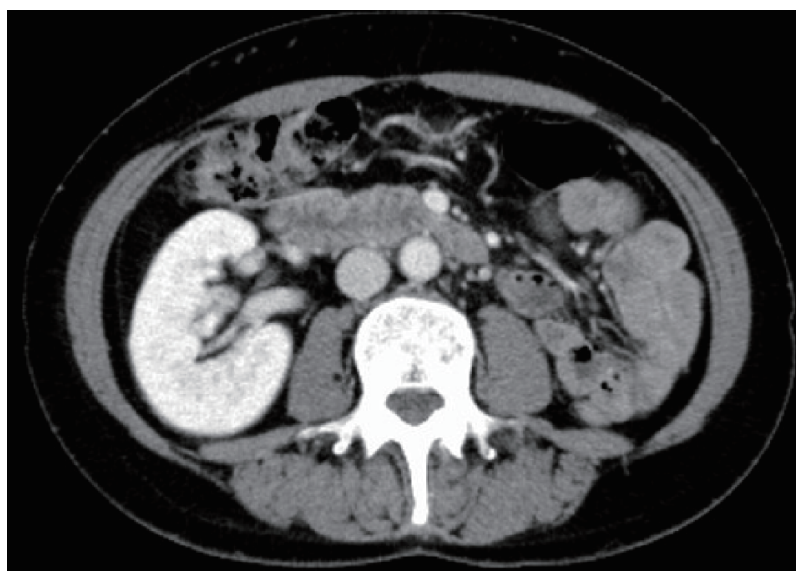

Figure 2. CT scan shows the absence of the left kidney. CT: computed tomography. left renal agenesis, uterine didelphys, and obstructive hemivagina, we diagnosed her with OHVIRA syndrome and CCC of the cervix and classified her as International Federation of Gynecology and Obstetrics stage IB1.

We performed an abdominal radical hysterectomy, including bilateral salpingo-oophorectomy and dissection of the bilateral pelvic lymph nodes. During the surgery, neither obvious effusion nor metastasis was observed in the abdominal cavity. We observed two corpora of the uterus in her pelvis. Her left ureter led to the left cervix, and the renal side of the left ureter ended at the bifurcation of the common iliac vessels; thus, her left ureter was a so-called ectopic ureter. We resected the ectopic ureter along with her left cervix and vaginal wall. A macroscopic examination of the excised specimens showed the presence of two cervixes, two corpora of the uterus, and a tumor measuring $1.0 \times 2.0 \mathrm{~cm}$ on the left cervix. Her left ectopic ureter led to the left cervix and opened in the endometrium (Fig. 3).

The bladder catheter was removed at postoperative day 5. The patient was discharged postoperative day 10 after an uneventful recovery. Following surgery, the patient received no treatment and had no evidence of disease 12 months after therapy completion. Her postoperative course was uneventful.

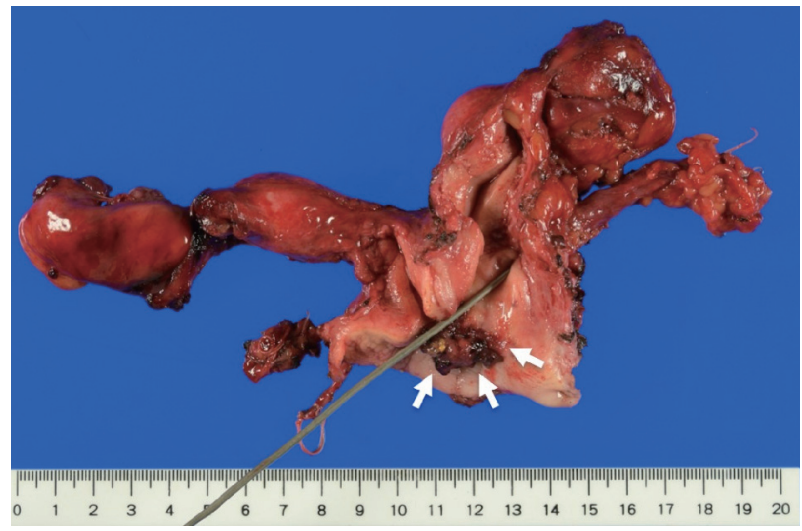

Figure 3. Surgical specimen shows a cervical tumor with uterus didelphys and her left ectopic ureteral orifice in the left obstructed hemivagina. A tumor is detected on the left side of the uterine cervix (white arrows). The surgical sonde was passed through the left ectopic ureter. 


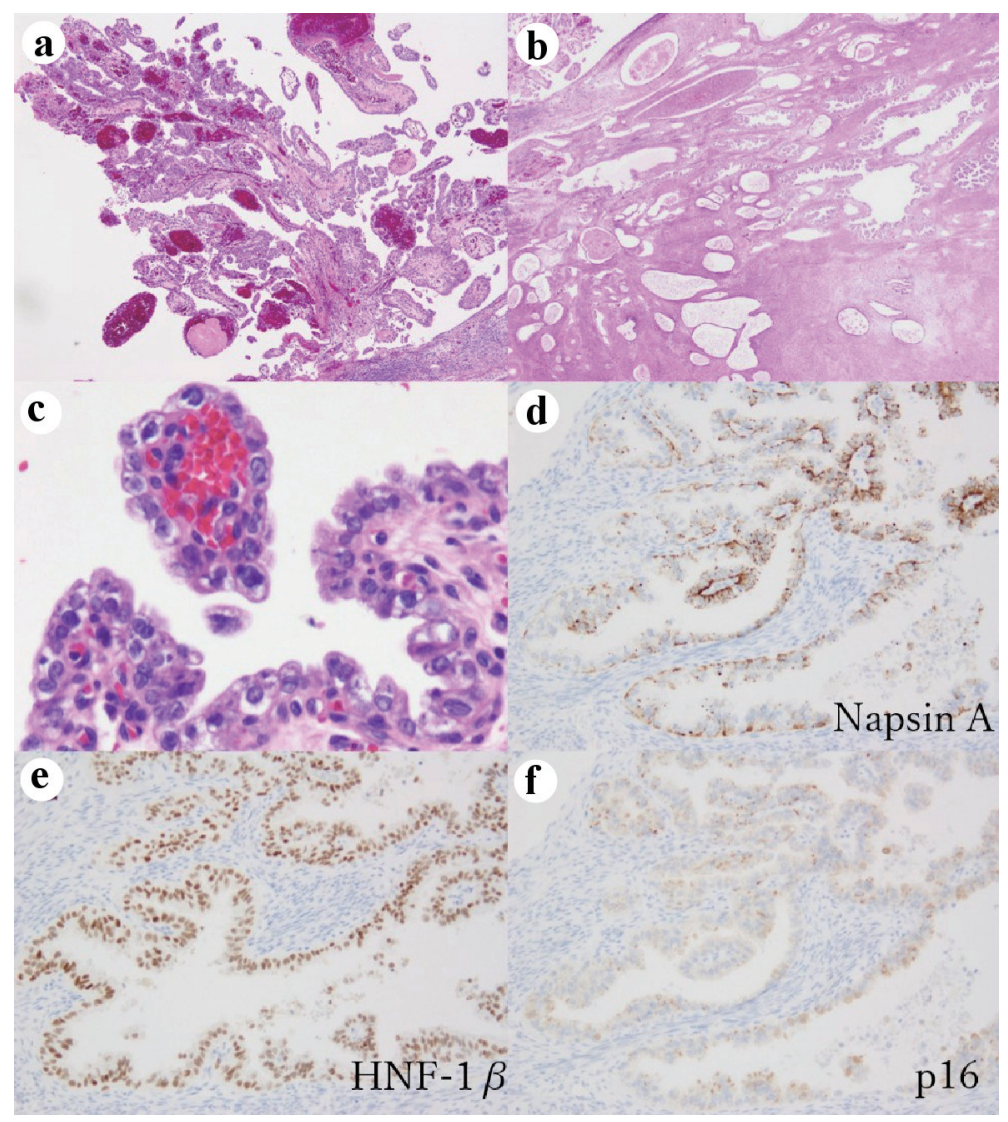

Figure 4. Histopathological findings of cervical clear cell carcinoma. At low-power view, the tumor shows papillary growth (a) and tubulo-cystic growth pattern (b) (hematoxylin and eosin stain (H\&E), $\times 40)$. At high-power view, tumor cells have enlarged nuclei with prominent nucleoli and clear cytoplasm (c) (hematoxylin and eosin stain (H\&E), $\times 400)$. Immunohistochemical staining show the tumor cells are positive for napsin A, $\times 200$ (d); and HNF-1 $\beta, \times 200$ (e); whereas p16 block positive pattern $(\times 200)$ is not observed in the tumor (f).

Postoperative histopathology revealed uterine cervical CCC, without invasion of the uterine wall, no lymph vascular invasion, and negative surgical margins. No metastatic lymph nodes on the 22 lymph nodes or invasion of the parametrium were observed, resulting in a tumor-node metastasis classification of pT1b1N0M0. The histopathological findings showed papillary growth and a tubulo-cystic growth pattern, which revealed CCC. Immunohistochemical staining showed that the tumor cells were positive for napsin A and HNF-1 $\beta$, while block positivity of p16 was not observed. Furthermore, the loss of PAX2 expression, retained ARID1A and PTEN expression, and wild-type pattern of p53 staining were observed (Fig. 4).

\section{Discussion}

The pathogenesis of OHVIRA syndrome is thought to be a result of the abnormal development of the Wolffian ducts and fusion failure of the Mullerian ducts, which leads to unilateral renal agenesis, imperforated hemivagina, and uterine didelphys and bicollis. The overall prevalence of Mullerian duct abnormality (MDA) is $2-3 \%$ [10]. The incidence of OHVIRA syndrome remains unknown, with few published case reports thus far. Very few reports are available regarding OHVIRA syndrome with cancer, particularly uterine cervical cancer [11]. Kaba et al also reported a case of cervical endometrioid adenocarcinoma diagnosed with OHVIRA syndrome [12]. To the best of our knowledge, our case of CCC of uterine cervical cancer associated with OHVIRA syndrome is the second such reported case [9].

Although the characteristic triad of this syndrome consists of didelphys uterus, obstructed hemivagina, and ipsilateral renal agenesis, a variety of uterine and renal anatomical variants have been reported $[7,8]$. Cases with dysplastic and atrophic kidneys might experience symptoms such as persistent vaginal drainage after resection of the vaginal septum. However, these variants are often ignored, possibly resulting in delayed diagnosis and treatment. Schlomer et al reported cases of symptomatic atrophic and dysplastic kidney with an ectopic ureter opening into an obstructed hemivagina. Such patients' conditions are not detected by any CT or MRI imaging modality and are often overlooked. Patients would suffer from symptoms such as persistent vaginal drainage after resection of the vaginal septum and would require additional treatment. That study raised awareness of these conditions, which can be challenging to diagnose, and insisted that clinicians be aware of 
this possibility [8]. There are an increasing number of reports of OHVIRA syndrome with ipsilateral dysplastic kidneys or ectopic ureters; such conditions do not fit the classic definition of OGVIRA syndrome $[4,13,14]$. In our case, we observed an ectopic ureteral orifice in the left obstructed hemivagina (Fig. 3 ). The absence of a ureteral orifice in the bladder by cystoscopy must be confirmed to diagnose these cases.

Zhang et al reported a subclassification system of OHVIRA syndrome: "3O" (obstruction, ureteric orifice, and outcome) [15]. They applied this system to existing case series to verify whether it is reasonable and effective, and they found that almost all anatomical variants associated with OHVIRA syndrome were incorporated into the system. They reported the instructive significance of this system for determining surgical strategies. Although a nephroureterectomy might be considered in a case of a dysplastic kidney with an ectopic ureter to preserve the possibility of pregnancy, we resected the uterus along with the vaginal wall and ectopic ureter considering the possibilities of cervical cancer infiltration. Pathologically, no malignant cells were observed in the surgical specimens including the resected parametrium, the vaginal wall, and the ectopic ureter.

Women with OHVIRA syndrome presenting as MDAs are generally diagnosed during the adolescent and reproductive period after the onset of menstrual bleeding. The clinical symptoms and findings of MDA vary widely and include irregular spotting, dysmenorrhea, cyclic pelvic pain, urinary incontinence, infertility, and difficulty with sexual intercourse, depending on the particular anomaly. Early diagnosis and timely treatment could relieve symptoms and prevent adverse obstetrics outcomes such as preterm birth, fetal growth retardation, and cesarean section. While there are scarce reports regarding obstetrics outcomes in women with OHVIRA syndrome, Albulescu et al reported two cases of good pregnancy outcomes with OHVIRA syndrome [16]. Rega et al reported that $2-4 \%$ of women with MDA had normal reproductive outcomes [17]. Although our patient presented with intermittent vaginal bleeding after menopause, she was nulligravida and did not report any symptoms until menopause.

If the tumor existed in the hemiobstructed vaginal septum, a preoperative diagnosis would be difficult. Kusunoki et al reported the difficulty of a preoperative diagnosis of OHVIRA syndrome with uterine cervical cancer. They chose to perform a total simple laparoscopic hysterectomy for a case of $\mathrm{OH}-$ VIRA syndrome with stage IB1 uterine cervical CCC because of the location of the cervical mass, which was inaccessible through the vagina [9]. In our case, the tumor was located on the center of the duplicated cervix, so we could access the tumor and easily diagnose it. However, at the first visit, there only appeared to be one cervix when viewed through the vagina. Through the preoperative CT showing a left renal analgesia, duplicate uterus, and hemivagina, the possibilities of MDA could be diagnosed.

Previous studies have reported that women who had prenatal exposure to DES were at an increased risk for CCC of the vagina and uterine cervix early in life [18]. DES was used to prevent miscarriage and other pregnancy complications from 1948 to the early 1970s. It has been estimated that $2-4$ million pregnant women in the USA were treated with DES or a similar synthetic estrogen such as dienestrol. In 1971, the use of DES in pregnant women was reported to be strongly associated with the development of CCC of the vagina and cervix in exposed daughters in their late teens or early 20 s $[19,20]$. Although only two case reports exist that are associated with $\mathrm{CCC}$ of the cervix and OHVIRA syndrome, neither of those cases had utero DES exposure, similar to our case.

\section{Conclusions}

To our knowledge, we describe the second case of OHVIRA syndrome with CCC of the cervix; this case underwent a radical hysterectomy, and the left ureter was found to lead to the left cervix and opened in the endometrium, resulting in a socalled ectopic ureter. In addition to the typical symptoms of OHVIRA including uterine didelphys, obstructed hemivagina, and renal agenesis, clinicians should bear in mind that several anatomical variants exist. Our case indicated those variants include an atrophic kidney with an ectopic ureter and obstructed hemivagina.

\section{Acknowledgments}

None to declare.

\section{Financial Disclosure}

None to declare.

\section{Conflict of Interest}

The authors have stated that they have no conflict of interest.

\section{Informed Consent}

Written informed consent was obtained from the subject of this case report prior to preparation of this manuscript. There is no information permitting to identify the patient.

\section{Author Contributions}

Dr. TN and Dr. SK collected the case details. Dr. MK wrote and reviewed the manuscript. Dr. MU, MI and TK reviewed the manuscript. Dr. AA participated in the management of this case and contributed to gathering clinical information. All authors read and approved the final manuscript.

\section{Data Availability}

The data supporting the findings of this study are available from the corresponding author upon reasonable request. 


\section{References}

1. Purslow CE. A case of unilateral haematokolpos, haematometra, and haematosalpinx. BJOG. 1922;29(4):643.

2. Herlyn U, Werner H. [Simultaneous occurrence of an open Gartner-duct cyst, a homolateral aplasia of the kidney and a double uterus as a typical syndrome of abnormalities]. Geburtshilfe Frauenheilkd. 1971;31(4):340-347.

3. Wunderlich M. [Unusual form of genital malformation with aplasia of the right kidney]. Zentralbl Gynakol. 1976;98(9):559-562.

4. Smith NA, Laufer MR. Obstructed hemivagina and ipsilateral renal anomaly (OHVIRA) syndrome: management and follow-up. Fertil Steril. 2007;87(4):918-922.

5. Acien P, Acien MI. The history of female genital tract malformation classifications and proposal of an updated system. Hum Reprod Update. 2011;17(5):693-705.

6. Troiano RN, McCarthy SM. Mullerian duct anomalies: imaging and clinical issues. Radiology. 2004;233(1):1934.

7. Yilmaz S, Yildiz AE, Fitoz S. Herlyn-Werner-Wunderlich Syndrome: sonographic and magnetic resonance (MR) imaging findings of this rare urogenital anomaly. Pol J Radiol. 2017;82:216-219.

8. Schlomer B, Rodriguez E, Baskin L. Obstructed hemivagina and ipsilateral renal agenesis (OHVIRA) syndrome should be redefined as ipsilateral renal anomalies: cases of symptomatic atrophic and dysplastic kidney with ectopic ureter to obstructed hemivagina. J Pediatr Urol. 2015;11(2):77 e71-76.

9. Kusunoki S, Huang KG, Magno A. Laparoscopic en bloc resection of a para-cervical cancer with OHVIRA syndrome. Taiwan J Obstet Gynecol. 2018;57(1):141-143.

10. Shavell VI, Montgomery SE, Johnson SC, Diamond MP, Berman JM. Complete septate uterus, obstructed hemivagina, and ipsilateral renal anomaly: pregnancy course complicated by a rare urogenital anomaly. Arch Gynecol Obstet. 2009;280(3):449-452.

11. Oka E, Chiyoda T, Iwata T, Yamagami W, Aoki D. Uterine cervical cancer associated with obstructed hemivagina and ipsilateral renal agenesis (OHVIRA) syndrome:
A case report and review of the literature. Gynecol Oncol Rep. 2020;34:100645.

12. Kaba M, Gungor T, Baser E, Ozdal B, Sirvan L. Cervical cancer in a patient with uterus didelphys and obstructive hemivagina, ipsilateral renal anomaly (OHVIRA) syndrome. Arch Gynecol Obstet. 2013;288(1):229-230.

13. Capito C, Echaieb A, Lortat-Jacob S, Thibaud E, Sarnacki $\mathrm{S}$, Nihoul-Fekete C. Pitfalls in the diagnosis and management of obstructive uterovaginal duplication: a series of 32 cases. Pediatrics. 2008;122(4):e891-897.

14. Kiechl-Kohlendorfer U, Geley T, Maurer K, Gassner I. Uterus didelphys with unilateral vaginal atresia: multicystic dysplastic kidney is the precursor of "renal agenesis" and the key to early diagnosis of this genital anomaly. Pediatr Radiol. 2011;41(9):1112-1116.

15. Zhang J, Zhang M, Zhang Y, Liu H, Yuan P, Peng X, Cao $\mathrm{Z}$, et al. Proposal of the $3 \mathrm{O}$ (Obstruction, Ureteric Orifice, and Outcome) subclassification system associated with Obstructed Hemivagina and Ipsilateral Renal Anomaly (OHVIRA). J Pediatr Adolesc Gynecol. 2020;33(3):307313.

16. Albulescu DM, Ceausescu AE, Sas LM, Comanescu MC, Constantin C, Tudorache S. The Herlyn-Werner-Wunderlich triad (OHVIRA syndrome) with good pregnancy outcome - two cases and literature review. Rom J Morphol Embryol. 2018;59(4):1253-1262.

17. Raga F, Bauset C, Remohi J, Bonilla-Musoles F, Simon C, Pellicer A. Reproductive impact of congenital Mullerian anomalies. Hum Reprod. 1997;12(10):2277-2281.

18. Herbst AL. Behavior of estrogen-associated female genital tract cancer and its relation to neoplasia following intrauterine exposure to diethylstilbestrol (DES). Gynecol Oncol. 2000;76(2):147-156.

19. Herbst AL, Ulfelder H, Poskanzer DC. Adenocarcinoma of the vagina. Association of maternal stilbestrol therapy with tumor appearance in young women. N Engl J Med. 1971;284(15):878-881.

20. Noller KL, Decker DG, Lanier AP, Kurland LT. Clearcell adenocarcinoma of the cervix after maternal treatment with synthetic estrogens. Mayo Clin Proc. 1972;47(9):629-630. 